Bol. Soc. Bot. México 49:41:60(1989)

\title{
Fotosíntesis y economía del carbono en plantas superiores
}

\author{
Alejandro E. CAstellanos V.$^{1}$
}

\begin{abstract}
RESUMEN. Uno de los aspectos más desarrollados e importantes de la fisiología ecológica de plantas es el estudio de la fotosíntesis y la economía del carbono en el medio natural. En este artículo se analizan los avances recientes en este campo y se relaciona este conocimiento con la interpretación de aspectos de crecimiento y arquitectura de plantas así como la relación de la fotosíntesis con el movimiento de los órganos vegetales. Se indican además las posibilidades futuras del campo.
\end{abstract}

\begin{abstract}
One of the subjects of physiological ecology which has reached the greatest development and practical importance is the study of photosynthesis and carbon economy in plants growing in the natural environment. In this paper the recent developments in this field are analysed emphasizing the relationship of the plant production physiology with growth and architecture and also with organ movement. The future developments in the field are discussed.
\end{abstract}

\section{INTRODUCCION}

La ecofisiología de plantas superiores tiene varias conotaciones e interpretaciones. En el sentido más amplio, ecofisiología trata de entender los aspectos morfológicos, anatómicos y fisiológicos determinantes de la distribución y abundancia de las plantas superiores. En esta exposición se hace un breve resumen de los aspectos generales más importantes de la respuesta fotosintética en plantas superiores, la integración de dicha respuesta para una hoja en términos de ganancia, pérdida y eficiencia de conversión de carbono utilizando unidades de tiempo ecológicamente significativas para el individuo.

Las áreas ecofisiológicas de fotosíntesis y balance de carbono tienen una influencia directa o indirecta sobre los diferentes parámetros de adecuación, crecimiento, sobrevivencia y reproducción, por lo que su interacción con otras disciplinas de la ecología resultará extremadamente fructífera. Algunas de las perspectivas y limitantes para el desarrollo en México de este campo de estudio serán también exploradas.

1 Centro de Ecología, Universidad Nacional Autónoma de México, Apdo. Postal 70-275, Delegación Coyoacán, 04510, México, D. F. 


\section{FOTOSINTESIS}

La fotosíntesis se puede definir como la capacidad de fijación de dióxido de carbono por las plantas para la elaboración de compuestos orgánicos, posteriormente utilizados en diversas funciones metabólicas. Fotosíntesis es pues un proceso central en la generación de materia seca pues provee los esqueletos de carbono y energía necesarios para sintetizar los productos orgánicos utilizados por las plantas en su metabolismo.

La fotosíntesis consiste de tres tipos de eventos: fotoquímicos, de caracter fisicoquímico propiamente, de difusión de $\mathrm{CO}_{2}$ y metabólicos en donde la energía química generada en el primero y el $\mathrm{CO}_{2}$ capturado en el segundo proceso son utilizados bioquímicamente en la síntesis de carbohidratos. Estos procesos se describen brevemente a continuación.

\section{Procesos fotoquímicos.-}

La mayoría de los procesos de la fotosíntesis son efectuados dentro de organelos especializados denominados cloroplastos. La estructura de los cloroplastos presenta un sistema de membranas especializadas denominadas tilacoides en cuyo lumen se efectuan los procesos fotoquímicos, y una matríz denominada estroma en donde se efectúan las reacciones de fijación de carbono. Inicialmente una porción de la energía lumínica fotosintéticamente activa (PAR $=400-700 \mathrm{~nm}$ ) es capturada en forma de fotones por pigmentos de clorofila estructurados en complejos de pigmentos conocidos como centros de reacción o fotosistemas. En plantas superiores existen dos tipos de fotosistemas ambos actuando en paralelo, denominados PSI con pico de absorción en $700 \mathrm{~nm}$ y PSII con absorción máxima a $680 \mathrm{~nm}$. La energía lumínica atrapada por los fotosistemas es canalizada en una serie de reacciones oxido-reductivas en cadena en los espacios intermembranosos de los tilacoides del cloroplasto. La energía reductora generada en este transporte de electrones de la fotosíntesis, es utilizada en la formación de equivalentes de reducción (reducción de nucleótidos de pirimidina, $\mathrm{NADPH}^{+}$), que posteriormente serán empleados en la reducción de $\mathrm{CO}_{2}$ en el estroma de los cloroplastos de las células del mesófilo.

El transporte de electrones se encuentra acoplado a la generación de un gradiente de protones del estroma al interior del sistema de membranas de los tilacoides con la consecuente síntesis de adenosin-trifosfato (ATP) (Foyer, 1987). La generación de ATP además de utilizar la vía no cíclica de transporte de electrones antes descrita, puede efectuarse también utilizando una vía cíclica de transporte de electrones en donde el flujo de electrones responsables de la reducción de ferredoxina son utilizados en la reducción de plastoquinona para generar de nuevo ATP.

La porción inicial de la respuesta fotosintética al flujo lumínico absorbido es lineal y representa la eficiencia cuántica (0a) en la fijación de $\mathrm{CO}_{2}$,

$$
0 \mathrm{a}=0 \mathrm{i} \cdot \mathrm{a}
$$


donde "0i" es la eficiencia cuántica de la hoja a la luz incidente y "a" es el coeficiente de absorción de la misma. Esta eficiencia es similar para plantas que poseen vías metabólicas de fijación de $\mathrm{CO}_{2}$ semejantes. A concentraciones normales de $\mathrm{CO}_{2}$ y $\mathrm{O}_{2}$, la eficiencia (0a) es de $0.05 \mu$ moles de $\mathrm{CO}_{2}$ fijado/ $\mu$ moles PAR absorbido (Ehleringer y Björkman, 1977). Existen diferencias en 0i debido a cambios en la estructura de las hojas, factores ambientales, vías metabólicas de fijación de $\mathrm{CO}_{2}$ que son discutidas más abajo.

Procesos bioquímicos.-

La reducción de $\mathrm{CO}_{2}$ a partir de equivalentes de reducción generados por el proceso de transporte de electrones no-cíclico se efectua a través de un complejo sistema enzimático en el que juega un papel primordial la enzima Ribulosa Bisfosfato Carboxilasa-Oxigenasa (RUBISCO) quien utiliza tanto $\mathrm{CO}_{2}$ y $\mathrm{O}_{2}$ como sustrato y su aceptor Ribulosa bisfosfato (RUBP). RUBP, un compuesto de cinco carbonos es utilizado como aceptor de $\mathrm{CO}_{2}$ en la producción de dos triosas-fosfato (ácido 3-fosfoglicérico). Las triosas fosfato, a través de una serie de reacciones y sustituciones dentro del ciclo reductor del carbono, son utilizadas en la regeneración del sustrato (RUBP) y en la formación de azúcares como producto de la fotosíntesis. La utilización de $\mathrm{O}_{2}$ como sustrato por RUBISCO dentro del ciclo fotorrespiratorio resulta en la formación de un compuesto de dos (fosfo-glicolato) y otro de tres carbonos (ácido 3-fosfoglicérico) (Figura 1). El ciclo fotorrespiratorio presente principalmente en las plantas con vía fotosintética C3 (veáse más abajo) no solo resulta en un drenaje de carbono sino que representa un elevado gasto energético. Así pues, las reacciones "oscuras", esto es que no requieren la intervención directa de la energía lumínica, de la fotosíntesis y de fotorrespiración se encuentran ligadas por la actividad de RUBISCO.

Procesos de difusión.-

La capacidad de fijación de $\mathrm{CO}_{2}$ por una hoja es debida a factores metabólicos intrínsecos (discutidos anteriormente) y a la disponibilidad de $\mathrm{CO}_{2}$. De acuerdo a la primera ley de Fick y la ecuación general de transporte (Jones, 1983), el flujo de $\mathrm{CO}_{2}$ al interior de las células del mesófilo (A) es proporcional al gradiente de concentración $\left(\triangle \mathrm{CO}_{2}\right)$ multiplicado por un factor de conductancia de la hoja al $\mathrm{CO}_{2}(\mathrm{~g}$ '), esto es:

$$
\mathrm{A}=\Delta \mathrm{CO}_{2} \cdot \mathrm{g}^{\prime}
$$

en donde $\triangle \mathrm{CO}_{2}$ es la diferencia en concentración de $\mathrm{CO}_{2}$ en el exterior $(\mathrm{Ca})$ y el interior de la hoja (Ci). La resistencia de la hoja al flujo de $\mathrm{CO}_{2}\left(r^{\prime}=1 / g^{\prime}\right.$, inverso de la conductancia) tiene varios componentes relacionados con la resistencia en la capa limitante (r'a), en los estomas (r's) y en el mesófilo (r'm). Sustituyendo los diferentes componentes de resistencia en (2), 


$$
\mathrm{A}=\triangle \mathrm{CO} 2 /\left(\mathrm{r}^{\prime} \mathrm{a}+\mathrm{r}^{\prime} \mathrm{s}+\mathrm{r}^{\prime} \mathrm{m}\right)
$$

Excepto por la última que esta relacionada con limitaciones bioquímicas, las dos primeras son resistencias físicas al transporte de gases.

El papel de los estomas en la regulación del flujo de agua durante la transpiración es bien conocido. De la ecuación (3) puede observarse que la resistencia estomática juega un doble papel al estar involucrada tanto en la difusión de gases al interior $\left(\mathrm{CO}_{2}\right)$ como al exterior $\left(\mathrm{H}_{2} \mathrm{O}\right)$ de la hoja, y que la vía del agua hacia el exterior y del dióxido de carbono al interior de la hoja son compartidos, excepto en lo que se refiere a r'm.

La presencia de resistencias al flujo de agua puede ser conocida facilmente por lo que su medición es necesaria para el cálculo de la resistencia al flujo de $\mathrm{CO}_{2}$ al interior de la hoja. La difusividad del flujo de agua (g) respecto a la de $\mathrm{CO}_{2}$ es 1.6 veces mayor debido al tamaño de sus moléculas,

$$
g^{\prime}=g / 1.6
$$

por lo que conocida la conductancia al flujo de agua en la hoja, la conductancia al flujo de $\mathrm{CO}_{2}$ puede ser corregida proporcionalmente. La restricción impuesta por la conductancia estomática al flujo de $\mathrm{CO}_{2}$ opera en tal forma que su efecto sobre la fotosíntesis es generalmente mínima pues se encuentra operando cerca del punto de transición entre la limitante estomática y la limitante por carboxilación (Wong et al., 1979).

\section{Diferentes vías metabólicas.-}

Las plantas superiores han desarrollado a través de su evolución distintas vías fotosintéticas en relación con el aprovechamiento de la energía generada durante el proceso fotoquímico y con la fijación de $\mathrm{CO}_{2}$ en diferentes ambientes. El tipo de vía metabólica más ampliamente representado en plantas superiores involucra la sola presencia de RUBISCO en la fijación del $\mathrm{CO}_{2}$ atmosférico para formar compuestos de tres carbonos (triosas-fosfato) por lo que a esta vía se le conoce como $\mathrm{C}_{3}$. Una importante característica bioquímica de RUBISCO es que puede actuar como aceptor de $\mathrm{O}_{2}$ al mismo tiempo que actua como aceptor de $\mathrm{CO}_{2}$, por lo que ambos sustratos compiten por el sitio activo en la enzima. Como consecuencia de la afinidad diferencial de la enzima por $\mathrm{CO}_{2}$ y $\mathrm{O}_{2}$ y de la mayor presión parcial de $\mathrm{O}_{2}$ en el interior de la hoja, la fijación de $\mathrm{O}_{2}$ por RUBISCO resulta en la producción de compuestos de dos carbonos y en la activación del proceso fotorrespiratorio. La liberación de $\mathrm{CO}_{2}$ durante la luz relacionada con la actividad de fijación de $\mathrm{O}_{2}$ por el aparato fotosintético se denomina fotorrespiración. Existen pues, dos procesos que resultan en la liberación de $\mathrm{CO}_{2}$ durante la luz y deben ser distinguidos por su caracter diferente, fotorrespiración y respiración en la luz asociada a la actividad mitocondrial. La producción de compuestos de dos carbonos durante la fotorrespiración involucra no solo la desviación de carbono fotosintético hacia actividades no fotosintéticas sino además un costo energético muy alto en la forma de equivalentes de reducción y ATP. 


\section{DIAGRAMA SIMPLIFICADO DE LOS CICLOS DE CALVIN Y FOTORESPIRATORIO}

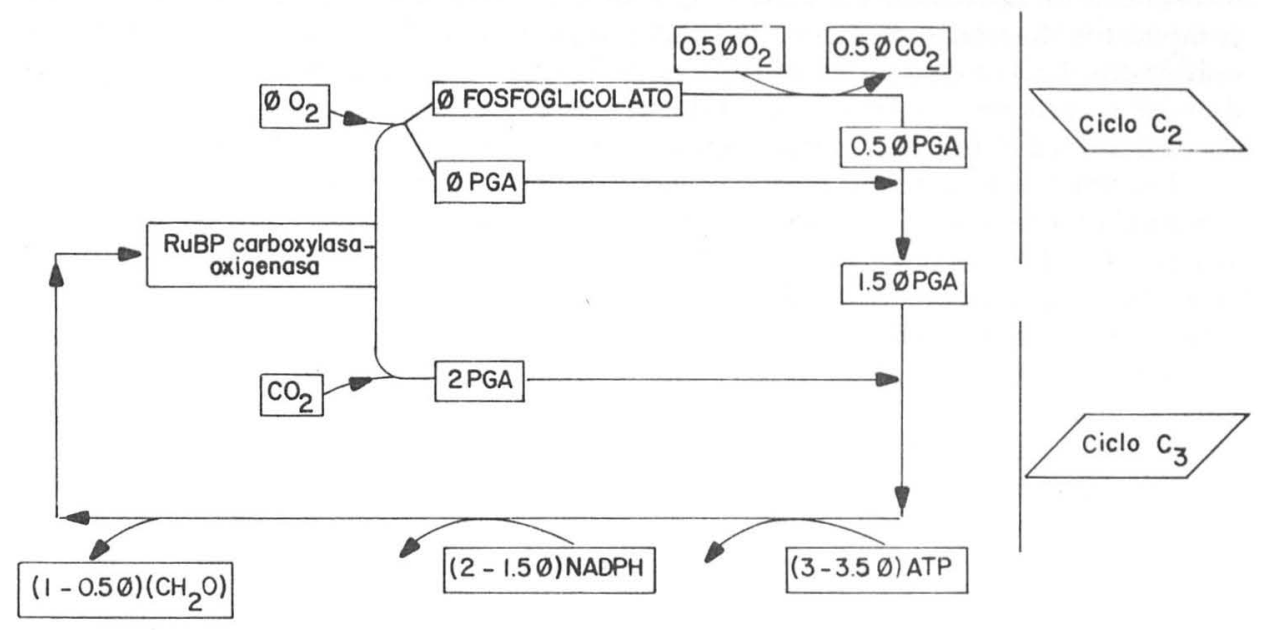

Figura 1. Diagrama simplificado de los ciclos de Calvin y fotorrespiratorio. El diagrama muestra las relaciones estequiométricas del número de carboxilaciones y oxigenaciones (de Farquhar et al., 1981).

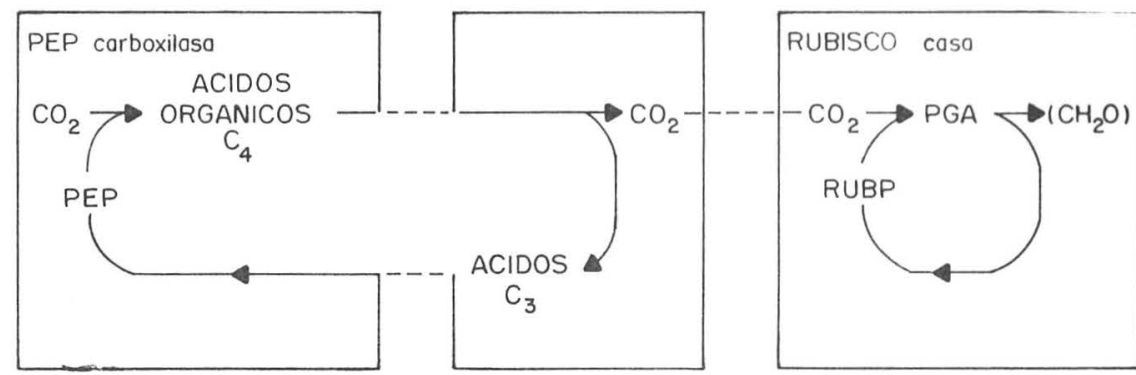

$\mathrm{C}_{3}$

MESOFILO

$\mathrm{C}_{4}$

MESOFILO

VAINA VASCULAR

Figura 2. Esquema de la distribución de los sistemas enzimáticos involucrados en las diferentes vías fotosintéticas (de Jones, 1983). 
La enzima fosfoenol-piruvato carboxilasa (PEPCASA) también actua dentro de la fotosíntesis en la condensación de $\mathrm{CO}_{2}$ y un compuesto de tres carbones para formar compuestos de cuatro carbonos utilizados posteriormente en la producción de carbohidratos. La afinidad de PEPCASA por $\mathrm{CO}_{2}$ para la formación de ácidos orgánicos de cuatro carbones, malato o aspartato, es mayor que la de RUBISCO por to que la descarboxilación de tales ácidos actua como mecanismo de concentración de $\mathrm{CO}_{2}$.

Existen dos tipos de vías fotosintéticas en las que existe un doble sistema enzimático durante la fotosíntesis con PEPCASA como la primera enzima fijadora de $\mathrm{CO}_{2}$, siendo siempre RUBISCO la responsable última de la fijación del $\mathrm{CO}_{2}$ fotosintético. En las especies conocidas como C4, PEPCASA se localiza dentro de células del mesófilo, espacialmente separada de RUBISCO localizada en el interior de las células del haz vascular (Figura 2). Esta distribución del aparato fotosintético dentro de las hojas da lugar a una estructura anatómica especializada característica denominada anatomía "Kranz". A diferencia de las especies C3, debido a la mayor afinidad de PEPCASA por $\mathrm{CO}_{2}$ y como consecuencia de su función concentradora de $\mathrm{CO}_{2}$ en el interior de las células, disminuciones en la concentración del $\mathrm{CO}_{2}$ atmosférico tienen poco efecto en la concentración de $\mathrm{CO}_{2}$ intercelular en las especies C4 (Osmond et al., 1982).

En las especies denominadas de metabolismo ácido crasuláceo (CAM, por Crasulacean Acid Metabolism), en que la primera enzima fijadora de $\mathrm{CO}_{2}$ es también PEPCASA, existe una separación temporal entre la actividad de PEPCASA para fijar $\mathrm{CO}_{2}$ realizada durante la noche y la actividad metabólica de síntesis de carbohidratos efectuada por RUBISCO durante el día. En las especies CAM, la apertura de los estomas durante la noche para la obtención de $\mathrm{CO}_{2}$ atmosférico, permite la disminución considerable de la pérdida de agua por lo que dicha vía fotosintética representa una estrategia adaptativa a ambientes desérticos y semidesérticos (Szarek y Ting, 1975).

Respuestas adaptativas del proceso fotosintético a limitantes ambientales.-

Los procesos fotosintéticos en las hojas se ven afectados de diversas maneras por factores ambientales y micro-ambientales. La respuesta de fotosíntesis a la temperatura se caracteriza por poseer un máximo en la capacidad fotosintética a temperaturas cercanas a la ambiental y tasas menores a temperaturas por debajo y por arriba de esta temperatura. El óptimo de temperaturas puede ser amplio como en el caso de un número de especies arbustivas y arbóreas de climas mediterráneo y desértico, o restringido como puede ser el caso de especies anuales desérticas (Ehleringer y Mooney, 1983). Los cambios en el óptimo de la respuesta fotosintética con temperatura están dados por diferencias en la difusión de $\mathrm{CO}_{2}$, en la velocidad de fotorrespiración y por la estructura de los lípidos en la membrana celular (Berry y Björkman, 1980; Pearcy y Ehleringer, 1984).

Diferencias en la humedad relativa ambiental representan diferencias en el déficit de presión de vapor de agua (VPD) para la hoja. Debido a que las plantas controlan su pérdida de agua a través de los estomas al mismo tiempo que utilizan éstos para la fijación de $\mathrm{CO}_{2}$, los mecanismos de regulación estomática en respuesta a diferencias en presión de vapor de agua pueden imponer un cierto control sobre la capacidad fotosintética de 
la hoja al afectar el gradiente de concentración de $\mathrm{CO}_{2}$ al interior de la hoja (ecuación 2). El efecto de la regulación estomática sobre la capacidad fotosintética optimiza la relación transpiración/fotosíntesis $(\delta \mathrm{E} / \delta \mathrm{A})$ (Cowan y Farquhar, 1977), esto es, el uso eficiente de agua (UEA = cantidad de agua perdida por unidad de carbón fijado) (Farquhar et al., 1980).

La importancia de la densidad de flujo fotónico para la fotosíntesis, dentro de la región fotosintéticamente activa $(\mathrm{PAR}=400-700 \mathrm{~nm})$, es manifestada por la gran cantidad de estudios efectuados sobre la aclimatación de las hojas e individuos a condiciones lumínicas contrastantes (Boardmann, 1977; Björkman, 1981). Las respuestas de las hojas e individuos a condiciones contrastantes de luz intensa y sombra han sido descritas extensamente bajo condiciones de laboratorio, sin embargo múltiples factores limitantes bajo condiciones de campo afectan las respuestas a la luz de manera menos predecible.

Castellanos y Mooney (no publ.) encontraron para el arbusto perennifolio Heteromeles arbutifolia, creciendo en condiciones naturales, que diferencias en la disponibilidad de recursos (luz, agua y nitrógeno) en diferentes años afecta el potencial de aclimatación de la especie a la luz (Figura 3). Es posible que varios factores limitantes sean optimizados simultáneamente durante el crecimiento del individuo (Bloom et al., 1985). La existencia de cambios en el potencial de aclimatación a la luz debido a diferencias en la disponibilidad de agua y nitrógeno ha sido caracterizado recientemente (Ferrar y Osmond, 1986; Clough et al., 1983). Lo anterior sugiere que la caracterización ecotípica en una determinada especie respecto a la respuesta de aclimatación a la luz debe ser cuidadosamente evaluada para determinar si se presenta tal aclimatación.

\section{ECONOMIA DEL CARBONO EN PLANTAS SUPERIORES}

La ecología evolutiva y la ecofisiología vegetal tratan de conocer las ventajas adaptativas en la asignación y eficiencia de uso de ciertos recursos en los individuos. En plantas superiores, la asignación de productos de la fotosíntesis (carbono, energía, fitomasa) a diferentes funciones como i)crecimiento, ii) reproducción, iii)mantenimiento (sobrevivencia) y iv) interacción con otros organismos (recompensa, defensa, etc.), es la resultante evolutiva desarrollada por el individuo en respuesta a condiciones ambientales particulares. De lo anterior se desprende que la selección natural deberá favorecer aquellas plantas cuya fisiología y arquitectura tiendan a maximizar su ganancia neta de carbono en un contexto competitivo particular (Givnish, 1986). Para lograr entender estos procesos adaptativos es necesaria la integración de las respuestas fotosintéticas en unidades de tiempo relevantes a los procesos involucrados en la economía del carbono.

Desde el punto de vista del balance de carbono, la síntesis de nueva biomasa (de una hoja por ejemplo) a partir de azúcares simples, requiere de la inversión de carbohidratos para proveer energía (necesaria para la adquisición, translocación y reducción de nutrientes) y para proveer esqueletos de carbono que puedan ser utilizados en componentes estructurales o funcionales (Figura 4). 


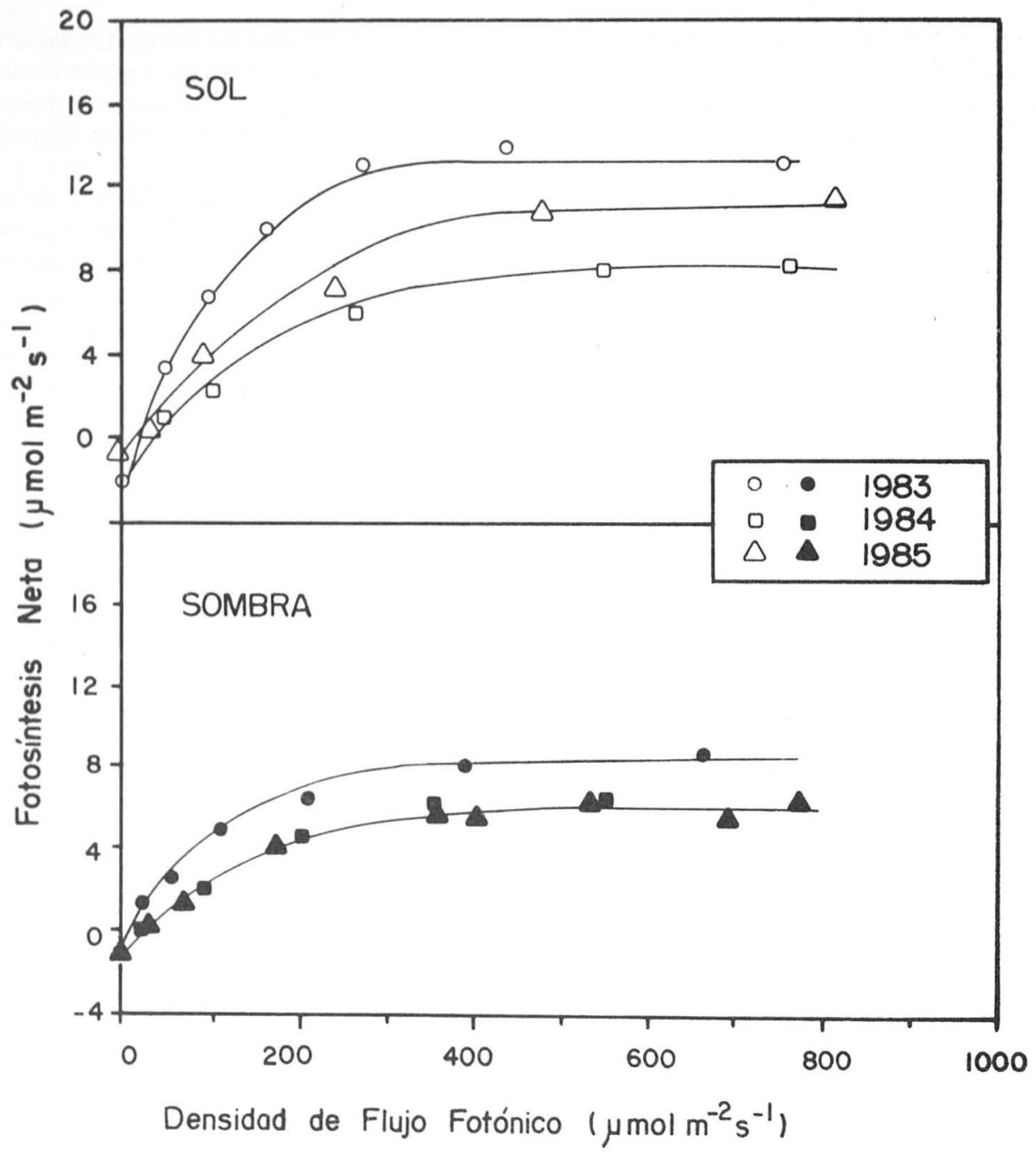

Figura 3. Variación inter-anual en el potencial fotosintético de aclimatación a la luz en la misma especie (Heteromeles arbutifolia) creciendo "in situ" bajo condiciones contrastantes de luz. 


\section{DE LA FOTOSINTESIS}

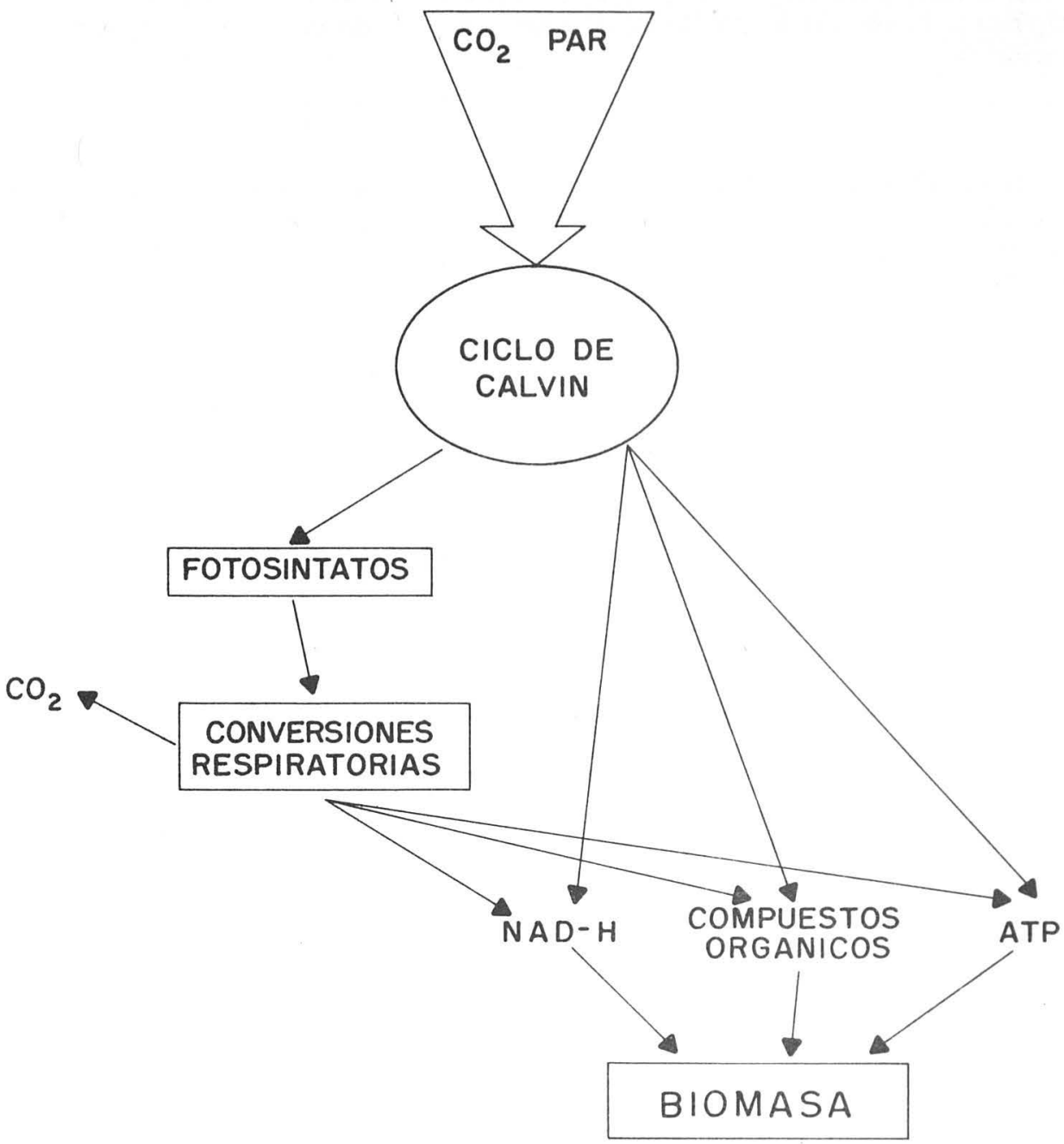

Figura 4. Diagrama esquemático de la gencración de energía, compuestos de carbono y biomasa a partir de la fotosíntesis. 
Parte de la energía generada y carbón liberado durante la respiración, es utilizado en funciones de crecimiento resultando en la incorporación de nueva biomasa (respiración asociada al crecimiento). La otra porción del componente respiratorio, respiración asociada al mantenimiento, es utilizada en el mantenimiento de la maquinaria metabólica (v.g., recambio de proteinas, flujo de iones, etc). De manera empírica se ha observado que la tasa de respiración (R) de un órgano (p. ej. hoja) es función de:

$$
R=a P+b W
$$

donde "P" es la tasa de fotosíntesis gruesa, "W" el peso seco del órgano y "a" y "b" son los coeficientes de crecimiento y mantenimiento, respectivamente (McCree, 1986). Esta relación puede ser expresada en base a cantidad de sustrato generado $(\Delta \mathrm{S} / \Delta \mathrm{t}) \mathrm{y}$ utilizado en ambas funciones,

$$
\Delta \mathrm{S}=\Delta \mathrm{SG}=\Delta \mathrm{SM}=(\Delta \mathrm{W}+\Delta \mathrm{SR})+\Delta \mathrm{SM}
$$

donde $\triangle \mathrm{S}$ es la cantidad de sustrato (en cualquiera de sus unidades, p.ej. carbohidratos, carbón, $\mathrm{CO}_{2}$, etc.) generado mediante la fotosíntesis, $\triangle \mathrm{SG}$ es el sustrato utilizado en el crecimiento y SM el sustrato utilizado en el mantenimiento (Thornley, 1977). Parte del sustrato utilizado en el crecimiento es usado en la incorporación de carbón en nueva biomasa $(\triangle W)$ y parte es respirado $(\triangle \mathrm{SR})$. De tal forma, la eficiencia de crecimiento (YG, la cantidad de materia seca producida por unidad de sustrato, está dada por:

$$
\mathrm{YG}=\Delta \mathrm{W} /(\Delta \mathrm{W}+\Delta \mathrm{SR})
$$

Sustituyendo entonces (6) y (7) en (5) se obtiene

$$
\mathrm{R}=(1-\mathrm{YG})-\Delta \mathrm{S} / \Delta \mathrm{t}+\mathrm{m}-\mathrm{Y} \bar{G}-\mathrm{W}
$$

en donde la tasa de respiración del órgano está dada en función de la eficiencia de conversión de sustrato a biomasa. Es necesario aclarar que el gasto respiratorio de crecimiento y mantenimiento, determinado mediante la ecuación 8 , no detecta gastos energéticos que no involucren pérdida de $\mathrm{CO}_{2}$ del órgano considerado o que se producen en otra parte del sistema y no son reflejados en la biomasa producida (Penning de Vries et al., 1974).

Para una misma cantidad de biomasa, el gasto de sustrato calculado a partir de glucosa puede ser mayor o menor en términos de costos de carbono. Tales costos, expresados como la cantidad de biomasa de cierta composición producida por un gramo de sustrato, (el valor de producción "PV" en Penning de Vries, et al., 1974), cambia dependiendo de la composición, esto es, del estado de reducción que posea el producto final. Si asumimos que toda la conversión de energía es realizada por sustrato presente dentro del mismo órgano, el costo de producción (PV) es aproximadamente equivalente a la eficiencia de crecimiento (YG) definida anteriormente (Thornley, 1977). Diferentes 
valores de eficiencia de producción ("YG") presentes en la conversión de glucosa a carbohidratos, lípidos, proteínas, etc., se presentan en la tabla 1.

La eficiencia de crecimiento (YG) varía en un gran número de especies y para diferentes órganos de la planta (Mooney y Gulmon,1982). Existe una tendencia a presentarse mayores eficiencias de crecimiento en partes aéreas y frutos comparados con raíces, aunque diferencias intra-específicas son halladas para un mismo órgano. Esto es particularmente evidente en el caso de YG para frutos en tomate y chícharo (Hole y Barnes, 1980; Walker y Thornley, 1977).

Valores altos de YG pueden estar relacionados con acumulación de compuestos menos costosos (carbohidratos), a diferencia de otros como lípidos, ligninas o compuestos nitrogenados. Pueden estar también relacionados con una importación neta (retranslocación) al órgano en cuestión en lugar de síntesis in situ de compuestos relativamente costosos (de alto peso molecular o nitrogenados). Ambas alternativas han sido exploradas por Sinclair y De Wit, (1975) quienes relacionaron los requerimientos de nitrógeno por unidad de fotosintato con valores de "YG" para semillas de veinticuatro especies cultivadas del cual se distinguieron cuatro grupos posibles. Un par de grupos con baja eficiencia de producción ("YG") debido a requerimientos elevados de sustrato en la síntesis de lípidos (oleaginosas) o de proteínas (soya). Otros dos grupos con "YG" relativamente elevados debido al menor estado de reducción de la biomasa(mayor proporción de carbohidratos en cereales), o retranslocación de compuestos altamente reducidos de la porción vegetativa a las semillas (legumbres de grano).

Los diferentes costos y mecanismos de asignación a estructuras reproductivas antes señalados no solo son importantes desde el punto de vista agronómico por sus consecuencias en el proceso productivo (Wilson et al., 1980), sino que tienen profundas implicaciones ecofisiológicas, ecológicas y evolutivas para la adecuación de los individuos. La retranslocación de nitrógeno para ser asignada a la producción de semilla es generalmente efectuada secuencialmente de las hojas más viejas a las más jóvenes (consecuentemente determinando la longevidad foliar), siendo las enzimas fotosintéticas uno de los primeros compuestos nitrogenados hidrolizados y retranslocados a las regiones de demanda (afectando así la capacidad fotosintética de las hojas). Este mecanismo para cubrir los costos incurridos durante la reproducción por el individuo es denominado "autodestructivo" por Sinclair y De Wit (1975) pues requiere la retranslocación de compuestos nitrogenados a partir de la porción vegetativa durante la fase de producción de semillas.

Para asegurar su defensa el individuo incurre en costos elevados de producción y mantenimiento de hojas (Merino et al., 1983) como es el caso de especies perennifolias o protegidas con concentraciones elevadas de compuestos secundarios de defensa. Coley et al., (1985) han propuesto que la disponibilidad de recursos en el habitat puede actuar como factor selectivo importante para la producción de compuestos secundarios (Figura 5). Mayor producción de compuestos secundarios de defensa bajo condiciones de "estrés" ha sido postulado por Grime (1979) y Coley et al., (1985). En condiciones de deficiencia en disponibilidad de nitrógeno se incrementa en algunas especies la presencia de compuestos clorogénicos al igual que condiciones de "estrés" hídrico. Igualmente, al incrementar la cantidad de luz roja sobre individuos de tabaco se ha logrado inducir la producción de alcaloides respecto a la de compuestos fenólicos, mientras que con- 
TABLA 1. Eficiencia de conversión de biomasa para diferentes compuestos orgánicos cuando la glucosa es tomada como el precursor. Las unidades son gramos de compuesto por gramo de glucosa empleada.

\begin{tabular}{lll}
\hline COMPUESTO & $\mathrm{Yo}^{*}$ & $\mathrm{Yo}^{\circ}$ \\
\hline Carbohidratos & 0.830 & 0.805 \\
Proteínas & 0.400 (en base a NO3 $)$ & 0.587 \\
Compuestos nitrogenados & 0.470 (en base a NO3 $)$ & \\
Lípidos & 0.330 & 0.322 \\
Lignina & 0.460 & 0.460 \\
Acidos orgánicos & 1.430 & 1.076 \\
& & \\
"Según varios autores. & & \\
${ }^{\circ}$ Según Penning de Vries y van Laar (1982) & \\
\hline
\end{tabular}


diciones de fotoperiodos de días largos incrementan la producción de fenoles y terpenos en algunas especies.

El análisis anterior permite visualizar algunas de las posibilidades de interacción entre diferentes campos de la biología vegetal que pueden darse mediante el estudio de la economía del carbono en plantas superiores. A continuación se discuten algunos ejemplos de procesos fisiológicos estudiados desde esta perspectiva particular.

Longevidad en las hojas.

Diversas hipótesis utilizadas en la explicación de las diferencias en longevidad de hojas han sido fundamentadas en términos de su balance de carbono (Chabot y Hicks, 1982), de optimización en la utilización de diferentes recursos como nitrógeno (Field, 1983; de Jong, 1982) o agua (Parkhurst y Loucks, 1972) y del costo energético necesario para su producción (Givnish, 1979; Bloom et al., 1985). La determinación del balance de carbono de las hojas provee de respuestas a las dos primeras hipótesis mientras que aspectos relacionados con la tercera hipótesis son discutidas más abajo.

Varios autores han encontrado una mayor actividad fotosintética en especies caducifolias que en especies perennifolias, sin embargo tales diferencias en actividad resultan en ganancias de carbono similares al ser integradas durante el tiempo de vida de la hoja (Karlsson, 1985). Al igual que Small (1972), diferentes autores han encontrado que la menor actividad fotosintética en especies perennifolias se encuentra relacionada con una mayor eficiencia de uso de nutrientes, sugiriendo que la presencia de especies perennifolias es favorecida en habitats con poca disponibilidad de recursos (Chabot y Hicks, 1982; Field et al., 1983).

Resultados similares han sido encontrados por Castellanos y Mooney (en prensa) para Heteromeles arbutifolia, una especie perennifolia viviendo en habitats lumínicos contrastantes. En el caso de Heteromeles, la actividad fotosintética en individuos de sitios sombreados y sitios con alta irradiación es similar, sin embargo hojas de individuos viviendo en lugares soleados poseen longevidades menores (2-3 años) que hojas de individuos de lugares sombreados (5-7 años). Las diferencias en longevidad foliar en dicha especie no son el resultado de elevadas tasas de actividad fotosintética (metabólica) como sugieren otros estudios. La presencia de actividades fotosintéticas similares asociadas a diferencias morfoestructurales y de disponibilidad de recursos (luz) resultan, al igual que en estudios anteriores, en ganancias de carbono similares al final de la vida de la hoja. Las ventajas adaptativas de las diferencias en longevidad fueron detectadas en dicho estudio al analizar la eficiencia de uso de los recursos más limitantes en los respectivos ambientes, así, en sitios sombreados donde la cantidad de luz recibida es diez veces menor que en el sitio expuesto, la eficiencia de uso de la luz fue mayor mientras que en éstos últimos la especie maximiza la ganancia de carbono por unidad de agua haciendo un uso más eficiente del agua disponible (Tabla 2). 
TABLA 2. Componentes del balance de carbono y transpiración a lo largo de la vida de una hoja. Los datos son para hojas típicas de individuos de Heteromeles arbutifolia creciendo bajo condiciones de sol y bajo condiciones de sombra en el campo.

\begin{tabular}{|c|c|c|c|}
\hline EDAD & GANANCIA & PERDIDA & TRANSPIRACION \\
\hline Años & $\begin{array}{l}\text { DIURNA } \\
\text { molCO}_{2} \mathrm{~m}^{-2}\end{array}$ & $\begin{array}{l}\text { NOCTURNA } \\
\text { molCO}_{2} \mathrm{~m}^{-2}\end{array}$ & $\mathrm{kmol} \mathrm{m}^{-2}$ \\
\hline 0 & 48.9 & 2.6 & 15.1 \\
\hline 1 & 26.5 & 1.5 & 6.0 \\
\hline 2 & 2.7 & 0.2 & 0.6 \\
\hline \multirow[t]{2}{*}{ TOTAL } & 78.1 & 4.2 & 21.7 \\
\hline & \multicolumn{2}{|c|}{ SOMBRA } & - \\
\hline 0 & 41.2 & 1.6 & 16.7 \\
\hline 1 & 29.2 & 1.2 & 11.8 \\
\hline 2 & 25.8 & 1.1 & 10.2 \\
\hline 3 & 5.4 & 0.2 & 2.2 \\
\hline 4 & 3.0 & 0.1 & 1.2 \\
\hline 5 & 2.6 & 0.1 & 0.9 \\
\hline 6 & - & - & -- \\
\hline TOTAL & 107.1 & 4.3 & 43.1 \\
\hline
\end{tabular}


FOTOSINTESIS Y ECONOMIA

Movimiento de organos.

Los movimientos de órganos, particularmente hojas, están comunmente presentes en plantas superiores de habitats con marcada estacionalidad o heterogeneidad espacial y temporal. Los movimientos foliares pueden agruparse en tres categorias diferentes: a) Movimientos de azimuth (heliotrópicos); b) Cambios en ángulo (respecto a la horizontal) relacionados con condiciones de gran demanda hídrica, balance energético desfavorable o intensidades lumínicas limitantes para la fotosíntesis; c) Ondulaciones foliares comunmente encontradas en pastos de climas secos o en perennifolias de climas fríos. Los movimientos diaheliotrópicos (formando ángulos perpendiculares a los rayos solares) son movimientos de azimuth (dirección) que comunmente conllevan cambios en ángulo foliar para permitir la mayor incidencia de los rayos solares sobre la lámina foliar. En el caso de movimientos paraheliotrópicos (ángulos foliares paralelos a los rayos solares), las hojas presentan cambios de orientación y ángulos de la lámina foliar para minimizar la cantidad de intercepción de radiación.

Existen una serie de hipótesis que tratan de explicar desde el punto de vista ecofisiológico las ventajas adaptativas relacionadas al movimiento foliar para- y diaheliotrópico. En especies con movimientos paraheliotrópicos se menciona como importantes las siguientes ventajas adaptativas: i) una disminución en la carga energética de las hojas al disminuir la temperatura foliar (Forseth y Teramura, 1986), ii) una disminución en el déficit hídrico al evitar, iii) una gran pérdida de agua por transpiración y por lo tanto iv) incrementar la eficiencia de uso de agua. Un incremento en la eficiencia de uso de agua también ha sido postulado en el caso de movimientos diaheliotrópicos al v) incrementar la superficie de captura de radiación PAR (fotosintéticamente activa) y vi) un aumento en las tasas fotosintéticas de manera que pueden vii) incrementar la ganancia de carbono por unidad de agua transpirada.

A pesar del conocimiento descriptivo tan amplio que existe en la literatura, las consecuencias ecofisiológicas de tales movimientos son aún poco entendidas y su estudio incipiente (McMillen y McClendon, 1979). Existen diferentes escalas de tiempo en las que dichos movimientos pueden estar beneficiando el funcionamiento de las plantas. En el caso de movimientos en respuesta a estímulos mecánicos y heliotrópicos el lapso de tiempo de respuesta va de segundos a unas cuantas horas. Además se ha observado que el número de especies anuales que presentan movimientos heliotrópicos dentro de una comunidad es inversamente proporcional a la duración de la época favorable de crecimiento (Ehleringer y Forseth, 1980). La relación es también válida para una misma comunidad y en el caso de anuales desérticas, un mayor número de especies heliotrópicos crecen durante el verano comparadas con las que lo hacen durante el invierno. El papel de especies con movimientos heliotrópicos dentro de comunidades tropicales, en particular de aquéllas con estacionalidad marcada, no ha sido aún explorado.

La importancia de cambios en el ángulo foliar en el caso de algunas especies tropicales ha sido relacionada con el mantenimiento de un balance energético favorable durante el día. En el caso de individuos de Croton creciendo en Chamela, Jalisco, cambios en los patrones de movimiento foliares estuvieron de alguna manera relacionados a la presencia de condiciones de estrés hídrico en la planta (Figura 6), 


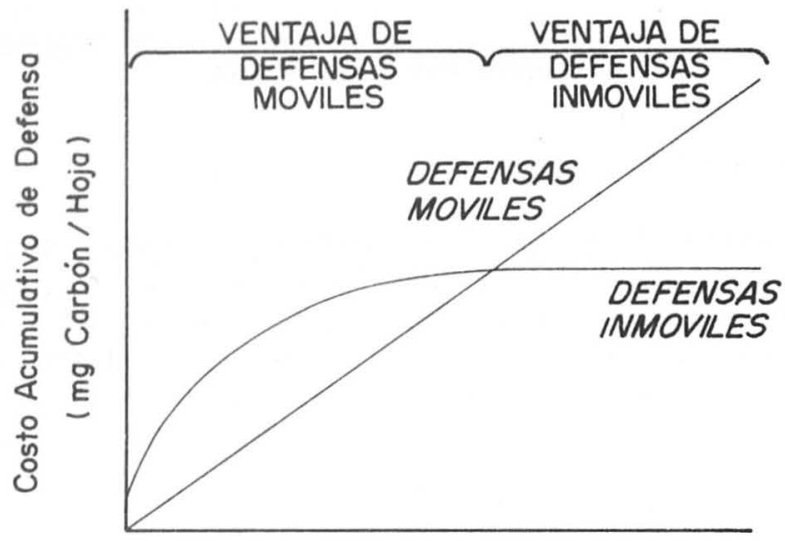

Periodo de Vida de la Hojo (meses)

Figura 5. Costo acumulado de la producción de compuestos secundarios de defensa en función de la presencia de compuestos nitrogenados o de carbono disponibles en las hojas. La longevidad foliar es en general función inversa a la presencia de altos contenidos de nitrógeno en las hojas (de Coley et al, 1985).
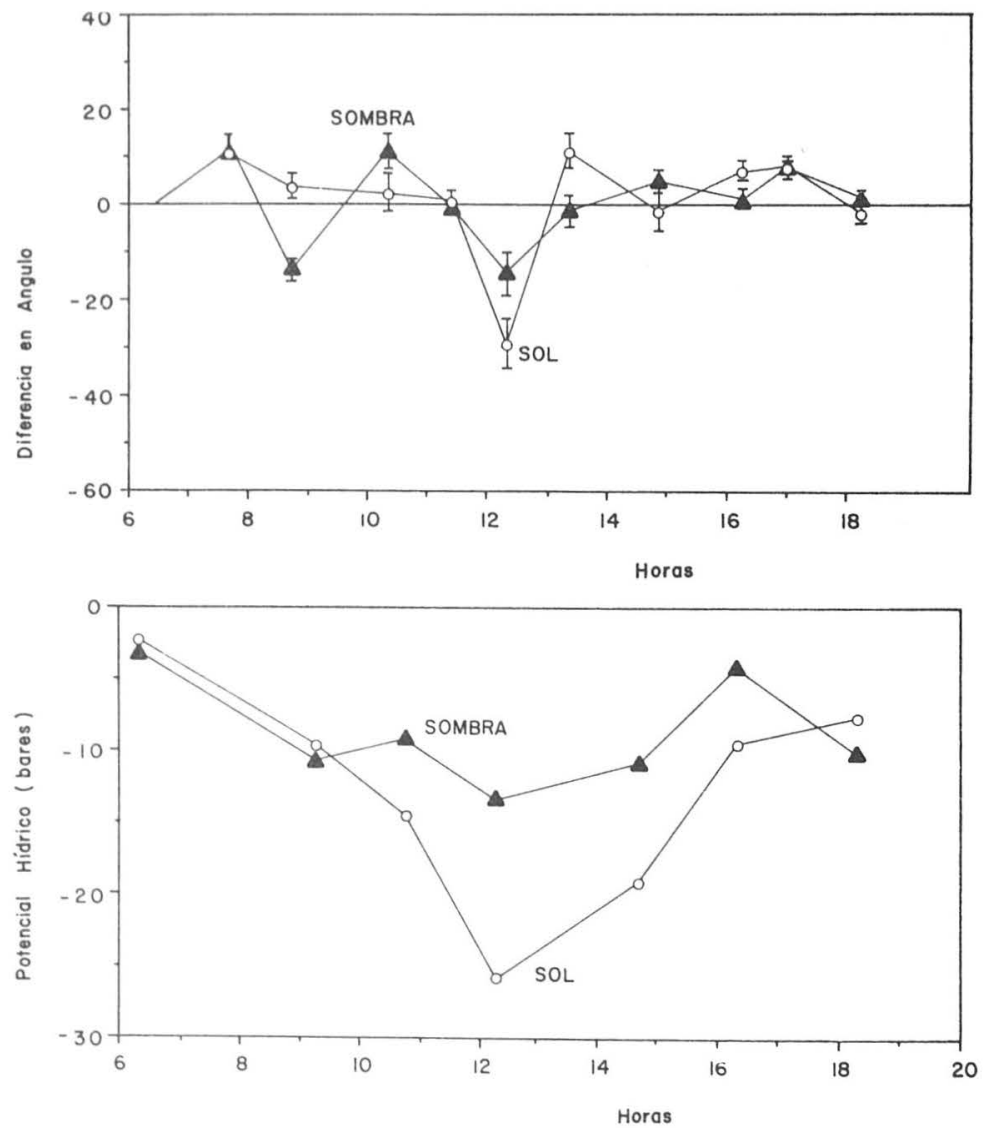

Figura 6. Cambios diurnos en el ángulo y potencial hídrico foliar de Croton niveus. Los datos son para individuos creciendo bajo condiciones naturales en Chamela, Jalisco. 
además cambios en el ángulo de las hojas pueden ser detectados de media a una hora después de ser irrigados. Individuos de Croton creciendo bajo diferentes condiciones de densidad de flujo fotónico (intensidad luminosa) tienen diferentes patrones de movimiento a lo largo del día.

Resulta obvio de lo anterior que definir la serie de mecanismos fisiológicos asociados al tipo de movimiento heliotrópico es crucial para entender el tipo de estrategia utilizado por las diferentes especies y poder así entender las limitantes fisiológicas en la distribución de las mismas, particularmente en el caso de especies en que ambos tipos de movimientos se suceden durante el transcurso del día. Cambios diurnos en el ángulo foliar en Vigna unguiculata siguen un patrón en el que diaheliotropismo se presenta en tempranas horas de la mañana y paraheliotropismo durante las horas de mayor temperatura e irradiación solar (Shackel y Hall, 1979). Bajo condiciones de invernadero, se ha encontrado en Phaseolus lunatus y Vigna unguiculata que los foliolos centrales difieren respecto a los laterales en sus movimientos foliolares aparentemente como resultado de diferencias en la capacidad fotosintética de los foliolos a estímulos de luz y probablemente de estado hídrico.

\section{PERSPECTIVAS FUTURAS Y DE INTERACCION.}

En su estudio la ecofisiología puede aplicarse a diferentes niveles de complejidad. En el caso de la fotosíntesis y el balance de carbono, es posible enfocarlos desde un punto de vista totalmente reduccionista al estudiar procesos a nivel de la hoja, o bien pueden ser enfocados a nivel del individuo o comunidad.

Una de las limitaciones en el avance de los estudios sobre fotosíntesis e intercambio de gases en el país es sin duda el alto grado de sofisticación en la tecnología requerida para realizarlos.

El estudio de los aspectos ecofisiológicos de la fotosíntesis es un campo sumamente vasto y interesante cuya importancia huelga decir es de la mayor importancia y múltiples son aún las preguntas que podemos formular en las que no conocemos su respuesta. Es posible mencionar tan solo a manera de una pequeña muestra en este espectro tan amplio que puede plantearse el ecofisiólogo dedicado al estudio de la fotosíntesis, el control ambiental de la misma y su integración con los demás mecanismos de crecimiento del individuo vegetal. En el caso de especies tropicales son aún pocos los estudios de los procesos antes me ncionados en el laboratorio y aún menor en número los realizados "in situ". Existen formas biológicas casi por completo olvidadas en este tipo de estudios como es el caso de las trepadoras (bejucos y lianas) tan abundantes dentro de las comunidades tropicales del país.

En las selvas tropicales perennifolias es común la apertura y cierre del dosel de manera más o menos frecuente (debido a daño físico o senescencia de individuos) y estacionalmente en las selvas deciduas o subdeciduas por las características de crecimiento de sus componentes dominantes. Diferentes aspectos de utilización de la luz por las especies dentro de este dinamismo de las comunidades son poco conocidos, como es el caso del efecto de la irregularidad lumínica de los rayos solares por su paso a través del dosel ("sunflecks"), o las diferencias en el potencial de aclimatación de la 
fotosíntesis a distintos ambientes lumínicos. En relación a la captura de luz, el estudio de los aspectos ecofisiológicos de la respuesta heliotrópica de las hojas seguramente aportará en el futuro una visión diferente sobre los mecanismos empleados por las plantas para utilizar de manera más eficiente los recursos disponibles (agua, nutrientes) al regular su metabolismo interno con respecto a las condiciones ambientales prevalecientes a su alrededor.

La vinculación del estudio ecofisiológico de la fotosíntesis con otros campos de la ecología están tan solo a la vuelta de la esquina. Una unión particularmente fértil será el estudio de los patrones de asignación de biomasa y energía con los estudios fisiológicos de costo-beneficio derivados mediante el análisis del balance de carbono en los individuos. La conjunción de ambos campos apenas ha sido explorada (Küppers, 1985) y las repercusiones en otras áreas de la ecología dedicadas al estudo de interacciones competitivas y estructuración de comunidades probablemente serán de gran importancia.

En el caso de nuestro país, el tipo de preguntas arriba esbozadas son igualmente válidas para casi cualquier tipo de especie y comunidad, ya sea de regiones tropicales o desérticas. Excepto utilizando métodos químicos en el caso de algunas especies CAM, hasta recientemente no había habido estudios de fotosíntesis en especies silvestres en el país. La importancia de los estudios sobre la fotosíntesis relacionando los procesos de asignación de biomasa y energía mediante el análisis del balance de carbono debería ser evidente de por sí. Tan solo por ejemplo el conocer las características fisiológicas de los procesos de generación de fitomasa en diferentes formas biológicas desérticas, pemitiría un conocimiento mecanístico mas preciso de los procesos involucrados en el crecimiento de estas especies en sus habitats naturales. La generación, a partir de este tipo de estudios, de conocimientos y técnicas aplicables dentro de la práctica agronómica convencional al cultivo de especies aprovechables ha sido la experiencia diaria en otros países durante décadas ya (Evenari et al, 1971; Schulze et al., 1975). Los estudios ecofisiológicos de las especies vegetales no solo pueden dar pautas para el desarrollo de una agricultura basada en la disponibilidad limitada de recursos, como es el caso en una gran parte del territorio mexicano ocupado por las zonas agrícolas de temporal del país, sino que son parte esencial para el enitendimiento de nuestros recursos silvestres.

\section{LITERATURA CITADA}

BERRY, J. y O. BJÖRKMAN. 1980. Photosyntetic response and adaptation to temperature in higher plants. Ann. Rev. Plant Physiol. 31: 491-543.

BJÖRKMAN, O. 1981. Responses to different quantum flux densities. pp. 57-107. En: Lange, O. L., P. S. Nobel, C. B. Osmond y H. Ziegler (Edrs.). Physiological plant ecology I. Encyclopedia of Plant Physiology. New Series. V. 12 A. Springer-Verlag, Berlin.

BLOOM, A. J., F. S. CHAPIN y H. A. MOONEY. 1985. Resource limitation in plants - An economic analogy. Ann. Rev. Ecol. Syst. 16: 363-392.

BOARDMAN, N. K. 1977. Comparative photosynthesis of sun and shade plants. Ann. Rev. Plant. Physiol. 28: 355-377. 


\section{FOTOSINTESIS}

CHABOT, B. F., J. F. CHABOT y D. J. HICKS. 1982. The ecology of leaf life spans. Ann. Rev. Ecol. Syst. 13: 229-259.

CLOUGH, J. M., J. A. TEERI y S. J. TONSOR. 1983. Photosynthetic adaptation of Solanum dulcamara L. to sun and shade environments. IV. A. comparison of North American and European genotypes. Oecología 60: 348-352.

COLEY, P. D., D. J. P. BRYANT y F. S. CHAPIN. 1985. Resource availability and plant antiherbivore defense. Science 230: 895-899.

COWAN, I. y G. D. FARQUHAR. 1977. Stomatal function in relation to leaf metabolism and environment. Symp. Soc. Exp. Biol. 31: 471-505.

DEJONG, T. M. 1982. Leaf nitrogen content and $\mathrm{CO}_{2}$ assimilation capacity in peach. J. Amer. Hort. Sci. 107: $955-959$.

EHLERINGER, J. y O. BJÖRKMAN. 1977. Quantum yields for $\mathrm{CO}_{2}$ uptake in $\mathrm{C} 3$ and $\mathrm{C} 4$ plants: dependence on temperature, $\mathrm{CO}_{2}$ and $\mathrm{O}_{2}$ concentration. Plant Physiol. 59: 86-90.

EHLERINGER, J. y I. FORSETH. 1977. Solar tracking by plants. Science 210: 1094-1098.

EHLERINGER, J. y H. A. MOONEY. 1983. Productivity of desert and mediterranean-climate plants. pp. 205-231. En: Lange, O. L., P. S. Nobel, C. B. Osmond y H. Ziegler (Edrs.). Physiological plant ecology I. Encyclopedia of plant physiology. New Series. V. 12 D. Springer-Verlag, Berlin.

EVENARI, M., L. SHANAN y N. TADMOR. 1971. The Negev challenge of a desert. Harvard University Press, Cambridge.

FARQUHAR, G. D., E. D. SCHULZE y M. KUPPERS. 1980. Responses to humidity by stomata of Nicotiana glauca $\mathrm{L}$. and Corylus avellana $\mathrm{L}$. are consistent with the optimization of carbon dioxide uptake with respect to water loss. Aust. J. Plant. Physiol. 7: 315-327.

FERRAR, P. y C. B. OSMOND. 1986. Nitrogen supply as a factor influencing photoinhibition and photosynthetic acclimation after transfer of shade-grown Solanum dulcamara to bright light. Planta 168: 563-570.

FIELD, C. 1983. Allocating leaf nitrogen for the maximization of carbon gain: Leaf age as a control on the allocation program. Oecologia 56: 341-347.

FIELD, C., J. MERINO y H. A. MOONEY. 1983. Compromises between water-use efficiency and nitrogen-use efficiency in five species of California evergreens. Oecologia 60: 384-389.

FORSETH, I. N. y A. H. TERAMURA. 1986. Kudzu leaf energy budget and calculated transpiration: the influence of leaflet orientation. Ecology 67: 564-571.

FOYER, C. 1987. Fotosíntesis. Compañía Editorial Continental, México.

GIVNISH, T. J. 1979. On the adaptative significance of leaf form. pp. 375-407. En: Solbrig, O. T., S. Jain, G. B. Johnson y P. H. Raven (Edrs.). Topics in plant population Biology. University of California Press, California.

GIVNISH, T. J. 1986. On the economy of plant form and function, Maria Moors Cabot Symp. 6th. Cambridge Univ. Press.Cambridge.

GRIME, J. P. 1979. Plant strategies and vegetation processes. John Wiley \& Sons, New York.

HOLE, C. C. y A. BARNES. 1980. Maintenance and growth components of carbon dioxide efflux from growing pea fruits. Ann. Bot. (London) 45: 295-307.

JONES, H. G. 1983. Plants and microclimate. Cambridge University Press, Cambridge. 
KARLSSON, P. S. 1985. Photosynthetic characteristics and leaf carbon economy of a deciduous and an evergreen dwarf shrub: Vaccinum uliginosum L. and V. vitis-idaea L. Holartic Ecology 8: 13-17.

KüPPERS, M. 1985. Carbon relations and competition between woody species in a Central European hedgerow. IV. Growth form and partitioning. Oecologia 66: 94-100.

MCCREE, K. J. 1986. Measuring the whole-plant daily carbon balance. Photosynthetica 20: 82-93.

MCMILLEN, G. G. y J. H. MCCLEDON. 1979. Leaf angle: an adaptative feature of sun and shade leaves. Bot. Gaz. 140: 437-442

MERINO, J., C. FIELD y H. A. MOONEY. 1982. Construction and maintenance cost of mediterraneanclimate evergreen and deciduous leaves I. Growth and $\mathrm{CO}_{2}$ exchange analysis. Oecologia 53: 208-213.

MOONEY, H. A. y S. L. GULMON. 1982. Constraints on leaf structure and function in reference to herbivory. BioScience 32: 198-206.

OSMOND, C. B., K. WINTER y H. ZIEGLER. 1982. Functional significance of different pathways of $\mathrm{CO}_{2}$ fixation in photosynthesis. pp. 480-547. En: Lange, O. L., P. S. Nobel, C. B. Osmond y H. Ziegler (Edrs.). Physiological plant ecology I. Encyclopedia of plant physiology (New Series Vol. 12-B). Springer-Verlag, Berlin.

PARKHURST, D. y O. L. LOUCKS. 1972. Optimal leaf size in relation to environment. J. Ecol. 60: 505-537.

PEARCY, R. W. y J. EHLERINGER. 1984. Comparative ecophysiology of C3 and C4 plants. Plant Cell \& Environment 7: 1-13.

PENNING DE VRIES, F. W. T., A. H. M. BRUNSTING y A. H. H. VAN LAAR. 1974. Products, requirements and efficiency of biosynthesis: A quantitative approach. J. Theoret. Biol. 45: 339-357.

SCHULZE, E-D., O. LANGE, U. BUSCHBOM, L. KAPPEN y M. EVENARi 1975. The role of air humidity and temperature in controlling stomatal resistance in Prunus armeniaca L. under desert conditions. III. The effect of water use efficiency. Oecologia 19: 303-314.

SCHACKEL, K. A. y A. E. HALL. 1979. Reversible leaflet movements in relation to drought adaptation of cowpeas Vigna unguiculata (L.) Walp. Austr. J. Plant Physiol. 6: 265-276.

SINCLAIR, T. R. y C. T. DE WIT. 1975. Photosynthate and nitrogen requirements for seed production by various crops. Science 189: 565-567.

SMALL, E. 1972. Photosynthetic rates in relation to nitrogen recycling as antaptation to nutrient deficiency in peat bog plants. Canad. J. Bot. 50: 2227-2233.

SZAREK, S. R. y I. P. TING. 1975. Physiological responses to rainfall in Opuntia basilaris (Cactaceae). Amer. J.Bot. 62: 602-609.

THORNLEY, J. H. M. 1977. Growth and maintenance respiration. A reinterpretation. Ann. Bot. (London) 41: 1191-1203.

WALKER, A. J. y J. H. M. THORNLEY. 1977. The tomato fruit: Import, growth, respiration and carbon metabolism at different fruit sizes and temperatures. Ann. Bot. (London) 41: 977-985.

WILSON, D. R., C. H. M. VAN BAVEL y K. J. MCCREE. 1980. Carbon balance of water-deficient grain sorghum plants. Crop Sci. 20: 153-159.

WONG, S. C., I. R. COWAN y G. D. FARQUHAR. 1979. Stomatal conductance correlates with photosynthetic capacity. Nature 282: 424-426. 